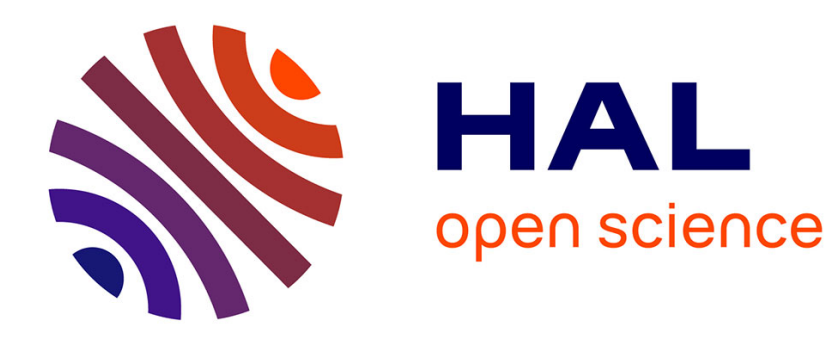

\title{
Fictions de l'énigme: vers une poétique du faux tableau
} Loïse Lelevé

\section{To cite this version:}

Loïse Lelevé. Fictions de l'énigme: vers une poétique du faux tableau. Revue de Litterature Comparee, 2016, Varia, 1 (357). hal-01867823

\section{HAL Id: hal-01867823 \\ https://hal.science/hal-01867823}

Submitted on 4 Sep 2018

HAL is a multi-disciplinary open access archive for the deposit and dissemination of scientific research documents, whether they are published or not. The documents may come from teaching and research institutions in France or abroad, or from public or private research centers.
L'archive ouverte pluridisciplinaire HAL, est destinée au dépôt et à la diffusion de documents scientifiques de niveau recherche, publiés ou non, émanant des établissements d'enseignement et de recherche français ou étrangers, des laboratoires publics ou privés. 


\section{Fictions de l'énigme : vers une poétique du faux tableau}

Peut-on lire un tableau ${ }^{1}$ ? A fortiori, peut-on écrire (sur) la peinture ? Ces questions ont été reposées avec acuité au $\mathrm{XX}^{\mathrm{e}}$ siècle, quand la critique de l'iconologie, telle qu'elle s'était développée sous l'influence notamment d'Erwin Panofsky, a incité historiens de l'art, philosophes et écrivains à repenser les rapports entre texte et image. L'iconologie s'était affranchie de la méthode biographique-historique héritée de Vasari comme de la pure étude formelle de la «surface» de l'œuvre pour accéder à son sens ${ }^{2}$. Mais cela au prix d'une «certitude que la représentation [... sait] traduire tous les concepts en images, toutes les images en concepts ${ }^{3} \gg$, et in fine, pour certains historiens de l'art comme Georges DidiHuberman, de la récupération de l'image dans un système discursif qui prétendrait en maîtriser le sens et détenir un savoir sur elle. Contre cette «tyrannie du lisible " ${ }^{4}$, se développe une contestation du primat d'outils et d'approches linguistiques pour aborder les œuvres ${ }^{5}$. Peinture et discours sont de plus en plus pensés dans un rapport d'altérité : la «position de l'art est un démenti à la position du discours », écrit Jean-François Lyotard dès $1971^{6}$; des logiciens comme Nelson Goodman ${ }^{7}$ remettent en cause toute stricte adéquation entre le signe linguistique et le « signe » pictural.

À cette crise d'un discours à prétention scientifique - celui de l'histoire de l'art et du savoir de l'œuvre qu'il entend délivrer -, à cette critique de toute approche discursive de l'œuvre, fait pendant, dans le champ littéraire, une entreprise de « réévaluation » par la fiction du discours historique qui se mue en véritable «combat critique ${ }^{8}$ ». À partir du second XIX ${ }^{\mathrm{e}}$ siècle en effet, et de manière déterminante au $\mathrm{XX}^{\mathrm{e}}$ siècle, la figure du critique écrivain, dont l'œuvre se nourrit de la simultanéité de la pratique de la fiction romanesque et de la critique d'art ${ }^{9}$, se renouvelle face à une professionnalisation des discours et des rôles (à l'historien de l'art celui de la production d'un discours scientifique; au journaliste spécialisé celui de la critique $^{10}$ ). Les romanciers construisent alors des fictions qui assument un positionnement autonome pour tenir, «en marge de l'histoire de l'art, un discours différent sur la peinture, mêlant registres fictionnel et référentiel ${ }^{11} »$. «Le $\mathrm{XX}^{\mathrm{e}}$ siècle $[\ldots]$ a été celui du sacre de

\footnotetext{
${ }^{1}$ Tous mes remerciements vont à Mme Véronique Gély et M. Bernard Vouilloux pour leurs conseils avisés et leur aide.

${ }^{2}$ Voir Sylvie Deswarte-Rosa, «Introduction», dans Sylvie Deswarte-Rosa (dir.), À travers l'image. Lecture iconographique et sens de l'œuvre, Paris, Klincksieck, 1994, p. 13 sq.

${ }^{3}$ Georges Didi-Huberman, Devant l'image. Question posée aux fins d'une histoire de l'art, Paris, Les Éditions de Minuit, « Critique », 1990, p. 11.

${ }^{4}$ Ibid., p. 25.

${ }^{5}$ Voir par exemple James Elkins, On Pictures and the Words That Fail Them, Cambridge, Cambridge University Press, 1998 ou Nadeije Laneyrie-Dagen, «Lire la peinture ? », Europe, Peinture et Littérature, n 933-934, janvier-février 2007, p. 227-246. À l'inverse, d'autres continuent de valoriser les outils linguistiques, par exemple Laurent Gerveraux dans Voir, comprendre, analyser les images, Paris, La Découverte, 1994. Le débat est loin d'être clos : Sylvie Deswarte-Rosa, parmi d'autres, maintient la nécessité d'une approche iconologique. ${ }^{6}$ Jean-François Lyotard, Discours, Figure, Paris, Klincksieck, 1971, p. 13.

${ }^{7}$ Nelson Goodman, Langages de l'art. Une approche de la théorie des symboles, trad. Jacques Morizot, Nîmes, Jacqueline Chambon, 1990 (édition originale : Languages of art, Indianapolis, Bobbs-Merrill, 1968).

${ }^{8}$ Voir Nella Arambasin, Littérature contemporaine et histoires de l'art. Récits d'une réévaluation, Genève, Droz, 2007 , p. 126.

${ }^{9}$ Voir Nicolas Valazza, Crise de plume et souveraineté du pinceau. Écrire la peinture de Diderot à Proust, Paris, Classiques Garnier, «Études romantiques et dix-neuviémistes », 2013.

${ }^{10}$ Voir Dario Gamboni, «Propositions pour l'étude de la critique d'art du XIX ${ }^{\mathrm{e}}$ siècle », Romantisme. Revue du XIXe siècle, $\mathrm{n}^{\circ} 71$, premier trimestre 1991, p. 10.

${ }^{11}$ Laurence Brogniez, «Du roman du peintre à la fiction critique. Sur quelques modalités de l'écriture (auto)biographique dans le champ littéraire contemporain. Autour de Jean-Jacques Salgon (Le Roi des Zoulous) et Maryline Desbiolles (Les Draps du peintre) », Les Cahiers du GRIT, t. 1, Olivier Odaert et Jean-Louis Tilleuil (dir.), Louvain-la-Neuve, 2011, p. 93.
} 
l'écrivain critique d'art. Les plus grands poètes et romanciers européens ont construit un pan entier de leur poétique à partir d'éblouissements artistiques dont ils ont rendu compte ${ }^{12}$. » La littérature se présente en effet comme «le moyen le plus adapté pour parler de peinture ${ }^{13}$ », dans la mesure où, à l'encontre d'un discours académique remis en cause, se répand l'idée que «toute description de tableau n'[est] elle-même qu'une création littéraire ${ }^{14}$ ». Sous la plume des écrivains, la peinture devient pleinement «[é]laboration de signes, mais non pas construction d'une signification assurée [...], qui fonde sa prétention au sens en même temps que son hétérogénéité irréductible à l'univers des textes ${ }^{15} \gg$. Le $\mathrm{XX}^{\mathrm{e}}$ siècle, comme le siècle suivant, est donc le moment d'une double prise de conscience : celle qu'aucun discours n'épuise les possibilités de sens d'un tableau mais qu'il recrée un objet autre, textuel, à côté de la toile ${ }^{16}$; celle que face aux possibilités de sens de la toile, la parole, et le discours littéraire en particulier, demeurent une nécessité pour que le spectateur s'approprie le tableau $^{17}$. L'inclusion ou la description de tableaux dans la fiction contemporaine recouvre donc une double fonction: l'instauration d'un rapport à l'art et à la peinture inédit et poétique $^{18}$, la mise à l'épreuve des potentialités du récit au regard du tableau ${ }^{19}$. Mais la rencontre entre peinture et littérature, dans le cadre privilégié de la fiction narrative, peut devenir rivalité mortelle : «Inscrivant le tableau dans son espace, l'écriture le détruit [...]. Mieux vaut accepter [...] que ce ne soit que perversement que se lise une écriture qui feigne de renvoyer à ces objets uniques, radicalement singuliers et autres que sont les tableaux ${ }^{20}$. » $\mathrm{Si}$ la description de tableau, au $\mathrm{XIX}^{\mathrm{e}}$ siècle, se situait encore dans l'héritage de l'ekphrasis antique $^{21}$, dans les siècles suivants l'insertion d'une toile, réelle ou fictive, dans une fiction se fait souvent interrogation oblique sur les pouvoirs d'un récit qui écrit la peinture pour mieux consacrer le triomphe de la fiction littéraire.

Ce basculement est particulièrement visible dans des œuvres qui mettent en scène un faux tableau, inventé par l'auteur pour les besoins de son récit, qui ne renvoie à aucun référent

\footnotetext{
${ }^{12}$ Pascal Dethurens, «Introduction. Les poètes de Zeuxis », dans Pascal Dethurens (dir.), Écrire la peinture. De Diderot à Quignard, Paris, Citadelles et Mazenod, 2009, p. 17.

${ }^{13}$ Ibid., p. 18.

${ }^{14}$ Ibid.

${ }^{15}$ Daniel Bergez, Littérature et peinture, Paris, Armand Colin, 2011, p. 72.

${ }^{16}$ Voir Bernard Vouilloux, La Peinture dans le texte. XVIII ${ }^{-}-X X^{e}$ siècles, Paris, CNRS Éditions, 1994, p. 89sq. : « À partir de cette rupture capitale que marquerait dans les arts plastiques la fin du XIX ${ }^{\mathrm{e}}$ siècle, ce ne serait plus la reproduction mimétique de la réalité qui procurerait sa légitimité à la peinture. [...] Coupée du sujet et donc de l'iconographie, et avec eux du substrat humaniste sur lequel reposait la théorie de la mimèsis comme imitation, la peinture se serait du même coup retranchée de tout ce qui en elle se prédisposait au langage. [...] Aussi [...] aurait-on l'intuition que la description du tableau ne peut plus compter sur les formules d'une tradition, mais s'inventer à chaque fois (dans) le langage que lui fait parler l'œuvre [...]. À la recherche des conditions qui la rendent possible, elle en viendrait à questionner le langage et sa limite, la peinture comme limite. »

${ }^{17}$ Voir Nadeije Laneyrie-Dagen, art. cit., p. 238sq. : « À l'aube du XXI ${ }^{\mathrm{e}}$ siècle, les conditions de l'analyse picturale ont évidemment changé. Il n'est plus guère en vogue de défendre que la ressemblance est l'objectif d'une œuvre d'art ou son efficacité narrative un critère d'appréciation. [...] Pour autant, face aux œuvres, la parole continue à s'élever [...]. "[L]'emboîtage" de commentaires demeure [...] la médiation presque inévitable par laquelle celui qui veut voir les peintures arrive à elles. »

${ }^{18}$ Voir Bernard Vouilloux, op. cit., p. 106 : «le discours [...] se donne comme contre-don symbolique s'échangeant à la donation de la peinture dans le tableau. Ainsi, le poétique, autre nom de cette auto-réflexivité, serait l'une des dimensions constitutives des discours modernes sur la peinture. »

${ }^{19}$ Ibid., p. 57 : «La description ne décrit plus des objets, mais le fonctionnement sériel du texte : elle en est la métaphore. »

${ }^{20}$ Jean-Pierre Guillerm, «Avant-propos », dans Jean-Pierre Guillerm (dir.), Récits/tableaux, Lille, Presses Universitaires de Lille, 1994, p. $14 s q$.

${ }^{21}$ Judith Labarthe-Postel, Littérature et peinture dans le roman moderne. Une rhétorique de la vision, Paris, L'Harmattan, 2002, p. 342 : «l'illusion consiste justement à faire croire [...] que ces mots sont une image, et possèdent les "pouvoirs de l'image" [...] à un degré bien plus grand qu'une simple image constituée de signes rhétoriques ; le texte tend alors vers autre chose que lui-même. »
} 
réel et se trouve inclus dans un texte reprenant les techniques des écrits scientifiques ou critiques sur l'histoire de l'art. Nous voudrions nous concentrer ici sur des textes qui cherchent à faire douter leurs lecteurs de l'existence même, hors de la fiction, de ces tableaux, dans la mesure où de tels textes nous semblent emblématiques de l'usage d'un discours fictif sur la peinture à des fins critiques ou métalittéraires. Ainsi, le narrateur des $O n z e^{22}$ de Pierre Michon prétend reconstituer le sens du tableau éponyme, sans jamais préciser qu'il s'agit d'un faux tableau ; et à travers son enquête sur la biographie du peintre François-Élie Corentin et sur les raisons troubles pour lesquelles le tableau, conçu comme un piège politique potentiellement meurtrier, lui a été commandé, il tente de donner un sens à une période historique, la Terreur, qui ne possède pas selon lui de tableau à sa mesure ${ }^{23}$.

Or, on trouvait déjà une telle construction narrative - qui mêle à la présence d'un faux tableau celle d'un schéma d'enquête tournant à la fois autour de la toile elle-même, de son auteur, mais aussi d'une énigme, d'un meurtre ou d'un piège auquel elle serait liée - dans $U n$ cabinet d'amateur ${ }^{24}(1979)$ de Georges Perec, dernier texte publié de son vivant, qu'on rapprochera du premier récit complet rédigé par l'auteur, entre 1957 et 1960, Le Condottière $^{25}$, deux œuvres qui mettent en scène une figure de faussaire. Dans le second récit, le faussaire Gaspard Winckler tue le commanditaire du tableau qui donne son titre à l'ouvrage en comprenant que sa tentative de créer un faux Messine authentique, non pas simple imitation mais véritable recréation a posteriori d'un tableau du peintre italien ${ }^{26}$, est vouée à l'échec parce que le tableau est tout sauf un ensemble de signes transposables sur une autre toile $^{27}$. Sa copie, qui s'en tenait aux marques visuelles immédiatement perceptibles de l'œuvre, s'avère insuffisante ${ }^{28}$. C'est en se lançant dans une complexe quête du sens de son meurtre qu'il pourra, peut-être, élaborer une relation fructueuse à la peinture. Le dernier récit publié de Perec est plus éloigné de ces préoccupations : il consiste avant tout dans la mise en place d'une mystification à tous les niveaux du texte, pour tromper personnages et lecteurs. Le tableau éponyme est à la fois l'instrument de la vengeance de son propriétaire Hermann Raffke contre les experts qui lui ont menti sur la valeur de ses tableaux, et un moyen de proposer une exploration ludique de l'histoire de l'art aux lecteurs du récit, qui multiplie à plaisir les descriptions incomplètes ou piégées et les fausses anecdotes historiques. L'enquête menée autour de la personnalité du peintre par le spécialiste Lester Nowak n'est qu'un moyen de plus pour entretenir le mystère, induire en erreur et remettre en cause la valeur des discours de savoir sur l'art. On retrouve aussi le lien entre faux tableau et meurtre dans un récit qui propose une structure d'enquête explicite, La tabla de Flandes (Le Tableau du Maître flamand $^{29}$ ), roman policier d'Arturo Pérez-Reverte, dans lequel il s'agit de déchiffrer l'énigme en latin cachée sous la surface du tableau La partida de ajedrez pour résoudre une série d'assassinats. QUIS NECAVIT EQUITEM ${ }^{30}$, qui donc a tué le chevalier qui, sur la toile, joue aux échecs avec le duc d'Ostenbourg ? Mais le récit détourne les codes du roman policier,

\footnotetext{
${ }^{22}$ Pierre Michon, Les Onze, [2009], Paris, Gallimard, « Folio », 2011.

${ }^{23}$ Voir Pierre Michon, Le Roi vient quand il veut. Propos sur la littérature, textes réunis et édités par Agnès Castiglione et Pierre-Marc de Biasi, Paris, Librairie Générale Française, 2007, p. 158.

${ }^{24}$ Georges Perec, Un cabinet d'amateur, [1979], Paris, Éditions du Seuil, « Points », 2001.

${ }^{25}$ Georges Perec, Le Condottière [1957-1960], Paris, Éditions du Seuil, « La librairie du XXI ${ }^{\mathrm{e}}$ siècle », 2012.

${ }^{26}$ Ibid., p. 58 : Winckler veut « réussir ce que jamais faussaire avant lui n'avait osé tenté : la création authentique d'un chef-d'œuvre du passé ».

${ }^{27}$ Voir Nelson Goodman, op. cit., p. 147 : «même les copies les plus exactes du tableau de Rembrandt sont de simples imitations ou contrefaçons de l'œuvre, non pas de nouveaux exemples. »

${ }^{28}$ Ibid., p. 56 : «Si représenter consiste à classer les objets plutôt qu'à les imiter, à caractériser plutôt qu'à copier, il n'est pas question d'enregistrer passivement. L'objet ne se pose pas tel un modèle docile, avec des attributs nettement séparés, et bien mis en évidence pour que nous l'admirions et en fixions l'image. »

${ }^{29}$ Arturo Pérez Reverte, La tabla de Flandes, [1990], Barcelone, Debolsillo, 2011, trad. Jean-Pierre Quijano, sous le titre Le Tableau du maître flamand, [1993], Paris, Librairie Générale Française, 2012.

${ }^{30}$ Ibid., p. 10 ; trad. cit., p. 8.
} 
puisque l'assassin - César, mentor de l'héroïne, Julia -, fait tout pour être découvert, et contribue même à la progression de l'enquête, qui s'avère double : retrouver la vérité sur le passé du duché d'Ostenbourg; comprendre qui, en s'inspirant des mouvements des pièces de la partie représentée sur le tableau, a tué Álvaro, l'amant de Julia. Pour Julia, et, sans doute, pour le lecteur, l'important est moins la solution que l'expérience à tirer quant à la manipulation des images et des textes : le récit joue sur la polysémie inhérente aux signes picturaux pour induire ses lecteurs en erreur.

Le récit contemporain, donc, loin de reprendre à son compte un éventuel constat d'échec du discours à appréhender le phénomène pictural, entend se poser comme un nouvel outil de compréhension de la peinture par la fiction et de la fiction par la peinture, tout en reconnaissant leur différence : dans ces textes, le tableau apparaît volontiers comme une énigme, une surface dont le sens ne se laisse pas percer. Tout est fait, semble-t-il, pour souligner la nécessaire incertitude de la lecture. Contre un discours de savoir s'établit une poétique ludique de l'enquête, dont les ambiguïtés interdisent toute position de certitude ; contre l'illusion d'une possible description transparente de l'œuvre, d'une adéquation du mot à l'image ${ }^{31}$, se construit un processus de mystification qui redéfinit les rapports du narrateur et du lecteur. La présence du faux tableau induit aussi, dans un contexte postmoderne de recyclage des grandes références culturelles, l'exigence d'un nouveau rapport aux œuvres érigées en « canons » incontournables.

\section{Figures de l'enquête}

L'enquête apparaît alors comme une nécessité appelée par la confrontation des personnages ou du narrateur au (faux) tableau. Cependant, incapable de percer le secret de la toile, elle s'avère rapidement être une construction narrative défaillante qui ne permet pas de saisir la vérité visée à travers elle.

\section{Du roman policier à l'enquête de fiction : le modèle et sa subversion ${ }^{32}$}

La tabla de Flandes se présente explicitement comme un roman policier : sa capacité à duper son lecteur vient du traitement qu'il réserve aux règles qui conditionnent l'écriture et la réception des œuvres de ce genre, et notamment à celles qui différencient roman à énigme et roman noir, tels qu'avait pu les définir Tzvetan Todorov dans Poétique de la prose ${ }^{33}$. Si, dans le premier cas, la diégèse vise à expliciter comment non seulement le crime, mais encore son récit et celui de l'enquête ont pu avoir lieu (le narrateur-observateur relatant l'histoire de son point de vue, sans agir directement), dans le second ce «n'est plus un crime antérieur au récit qu'on nous relate, le récit coïncide avec l'action [...]. La prospection se substitue à la rétrospection $^{34} \gg$. Or, tout est fait pour présenter le récit d'A. Pérez-Reverte comme un roman à énigme classique - le mot apparaît dès la première phrase de l'ouvrage : "Une enveloppe cachetée est une énigme qui en renferme d'autres ${ }^{35}$. » Le tableau, objet ancien, se présente

\footnotetext{
${ }^{31}$ Voir Louis Marin, «Mimèsis et description », dans De la représentation, Paris, Gallimard-Seuil, «Hautes Études », 1994, p. 253 : «Autrement dit, le désir de transparence des images aux choses et des noms aux images dénierait un trouble central, radical dans l'espace même du même où paraissaient s'accomplir les équivalences entre les unes et les autres. ». C'est sur ce déni de l'absence de transitivité entre la représentation picturale et son référent et entre la description et son objet, que vont jouer les romanciers pour mystifier les lecteurs.

${ }^{32}$ Sur le rôle particulier du roman policier et des «formes policières du roman contemporain » dans la réévaluation des discours sur l'art, voir notamment Nella Arambasin, op. cit, chapitre III.

${ }^{33}$ Tzvetan Todorov, Poétique de la prose, Paris, Éditions du Seuil, 1971.

${ }^{34}$ Ibid., p. 60.

${ }^{35}$ Arturo Pérez-Reverte, La tabla de Flandes, op. cit., p. 9 : «Un sobre cerrado es un enigma que tiene otros enigmas en su interior »; trad. cit., p. 7.
} 
comme le récit codé, sur un mode pictural, d'un crime antérieur, historique, qu'il faut résoudre. Les détectives - Julia, César qui cache son jeu, et leur adjuvant, Muñoz - sont des spectateurs extérieurs aux événements, et le récit relate l'histoire du déchiffrement du tableau. Le but est de faire oublier au lecteur l'avertissement qui ouvrait le livre : l'énigme n'est pas unique. La surface picturale est perçue comme le prétexte à une investigation rétrospective attitude qui correspond à la tradition historique héritée de $\operatorname{Vasari}^{36}:$ l'interprétation du sens du tableau semble tributaire d'une tentative de reconstitution de son histoire et de ses conditions de production. Mais le tableau est l'espace d'une temporalité complexe, pluridirectionnelle: son interprétation peut s'orienter vers le présent, et même vers l'avenir : la partie d'échecs qui donne son titre au tableau et qui devait révéler l'identité de l'assassin du chevalier devient l'outil de figuration et de prédiction de meurtres à venir. Le récit bascule vers le roman noir : l'héroïne est en danger de mort, l'issue du jeu fatal entre le mystérieux assassin et les autres personnages devient très incertaine. Le narrateur piège donc le lecteur en opérant une subtile confusion des genres et des structures narratives.

Le Condottière réutilisait déjà certaines structures du récit à énigme, quoique différemment : il y a bien une bipartition dans le roman, entre histoire du crime d'une part, histoire de son récit de l'autre. Seulement, l'histoire du crime n'est pas à reconstituer à partir de traces ou d'indices : elle est d'emblée donnée, dans tous ses détails. Le récit du meurtre vise bien son élucidation, mais il ne s'agit plus de poser la question du comment, mais celle du pourquoi: l'investigation devient psychologique. Le tableau est le déclencheur d'une crise chez Gaspard, relatée dans la première partie, questionnée dans la seconde. L'enquête ne cherche pas à résoudre une énigme dans le sens traditionnel du terme - un problème posé dont il faut trouver la solution, accompagné des éléments destinés à rendre possible la découverte de celle-ci. L'investigation, au contraire, s'attaque à ce qui échappe à toute réponse fixe - les motivations profondes d'un acte. La bipartition classique du roman à énigme se voit ici détournée de son propos original : le rôle du lecteur n'est plus directement de percer à jour un secret mais de percevoir de l'indicible.

La réflexivité intrinsèque et la temporalité double - entre rétrospection et prospection - de la trame classique du récit à énigme sont le fondement de jeux littéraires qui interrogent le rôle du lecteur ${ }^{37}$. Le tableau remet en jeu des règles génériques établies et fait systématiquement déraper l'enquête. Le ludisme inhérent au récit à énigme est réinvesti dans des narrations qui font du processus de lecture une tâche inquiétante et sans fin. La clôture du récit policier rassurait: l'ouverture systématique du sens rend les œuvres troublantes ${ }^{38}$. Comme le rappelle Isabelle Dangy-Scaillierez ${ }^{39}$, le schéma de l'enquête présente des aspects paradoxaux : élevant la rationalité et l'observation comme outils suprêmes de la découverte du vrai, l'enquête implique cependant la capacité de jouer avec le virtuel, de construire des récits possibles. Elle n'existe que dans un processus de mise en récit - «faire parler » les indices, construire un contenu discursif à partir de traces, lire des signes. Mais parce qu'aucun

\footnotetext{
${ }^{36}$ Voir Sylvie Deswarte-Rosa, op. cit., p. 12sq., pour qui l'approche historique et biographique « restera sans doute toujours une valeur sûre ».

${ }^{37}$ Voir Thomas Narcejac, Une machine à lire : le roman policier, Paris, Denoël/Gonthier, 1975, p. 95 :

« Freeman jouait en compagnie de son lecteur, contre le criminel. Il n'aurait pas imaginé qu'on pût jouer, en compagnie du criminel, contre le lecteur. »

${ }^{38}$ En cela, les textes que nous étudions relèvent aussi des «formes policières du roman contemporain », ces «écritures qui conjuguent l'évidence du soupçon et le besoin du jeu, le goût de l'intrigue - y compris jusque dans son inachèvement et le sentiment toujours plus fort que persistent, au-delà de la résolution, les énigmes » pour « poser certaines questions sur le sens, le signe, l'écriture, l'absence ou l'errance » (Denier Mellier et Gilles Menelgado, «Avant-propos», dans Denis Mellier et Gilles Menegaldo (dir.), Formes policières du roman contemporain, La Licorne nº44, Poitiers, Faculté des lettres et langues de l'Université de Poitiers, 1998, p. 6 et p. 5).

${ }^{39}$ Voir Isabelle Dangy-Scaillierez, L'Énigme criminelle dans les romans de Georges Perec, Paris, Honoré Champion, 2002, p. $92 s q$.
} 
discours n'épuise les possibilités de sens d'un tableau, l'enquête, même quand sa solution semble fermée, se présente comme une construction discursive qui ne parvient pas à atteindre la vérité recherchée par les personnages, que ce soit celle de leur identité ou du secret que renferme le tableau.

Dans le roman d'A. Pérez-Reverte, l'enquête s'avère construite vers l'avenir, vers l'initiation de Julia : César tente à travers ses meurtres de faire d'elle une femme émancipée de sa tutelle. Le savoir recherché se situe hors de l'enquête policière : il ne s'agit pas vraiment pour Julia de découvrir qui a tué le chevalier, ou Álvaro, mais d'apprendre à s'assumer en tant qu'être indépendant - vaste projet, impossible à résumer dans le contenu d'une solution d'enquête policière. La présence du tableau introduit, ou renforce, la gratuité de l'investigation et des réponses qui sont apportées. Certes, le tableau pose une question, doublement chiffrée : masquée, et écrite en latin. Mais la solution proposée est d'ordre romanesque : après avoir résolu la partie d'échecs qui y est représentée, Julia laisse cours à sa rêverie pour reconstituer les motivations et le sens des actions des personnages, dans une vision hallucinée de la scène de crime passée ${ }^{40}$. Contre la solution rationnelle traditionnelle du roman policier, où chaque détail de la reconstitution du crime se voit justifié par un indice ou un impératif logique, la réponse à la question posée par le tableau est nettement marquée par la rêverie et le fantasme. Plus que de mettre à jour la vérité du passé, il s'agit d'élaborer un récit possible des événements : le désir qui sert de moteur à l'enquête, en fin de compte, est un désir de fiction. De même, la découverte de la culpabilité de César importe peu : le discours du meurtrier sur son acte est bien plus riche d'enseignements potentiels que la découverte de son identité. L'enquête n'est qu'un prétexte à l'exploration d'identités : celle de César, de son passé, de son psychisme ; celle, à construire, de Julia, en tant que femme adulte et affranchie de sa fascination pour son mentor. Le roman est construit pour aboutir non à une solution mais à une révélation et l'enquête, dont les visées premières sont détournées de la recherche d'une vérité purement factuelle, se fait l'instrument d'une investigation esthétique, philosophique, psychologique.

Comme dans La tabla de Flandes, dans Le Condottière, le mouvement de l'enquête s'inverse. Si, traditionnellement, le détective est un lecteur dont le rôle est de déchiffrer des signes pour mettre au jour un sens préexistant, dans le roman de G. Perec, la quête de Gaspard est tout autre : le sens n'est pas à retrouver, il est à produire. «Tu crois que ça a un sens de commettre un meurtre ? Eh bien non. [...] Tu ne pouvais que te tromper ${ }^{41}{ }^{\prime}$, monologue le faussaire. La confusion des deux figures du détective et de l'assassin en un seul personnage assigne à Gaspard et à César le rôle d'exégète de leur propre action. L'acte criminel n'est pas constitué en énigme : ce qui doit être objet de savoir, ou plutôt de discours, est le sens à lui donner, la perspective dans lequel le replacer. Car au fond, la question du pourquoi, trop vaste pour songer sérieusement à y répondre, n'est pas essentielle; seule compte vraiment la suivante : comment mettre en récit l'acte ${ }^{42}$, le replacer dans un discours logique et cohérent, qui, sans épuiser ses possibilités de signification, permette à son auteur de se l'approprier, de l'inclure dans son histoire personnelle ? La mise en récit pourtant n'est jamais satisfaisante ; c'est ce que symbolise le tableau $^{43}$, à la fois support de la révélation et de la crise ${ }^{44}$, et figure

\footnotetext{
${ }^{40}$ Arturo Pérez-Reverte, op. cit., p. 184 sq., traduction p. $154 s q$.

${ }^{41}$ Georges Perec, Le Condottière, éd. cit., p. 50.

${ }^{42}$ Ibid., p. 43 : «Qu'y avait-il de trouble ? Quelque chose qu'il n'arrivait pas à comprendre. Un enchaînement, un lien. Un maillon. » La mise en récit a pour vocation de redonner une perspective cohérente à l'événement.

${ }^{43}$ «Los contornos son imprecisos, los matices cuentan. [...] La vida es une aventura incierta en un paisaje difuso », Arturo Pérez-Reverte, op. cit., p. 375 ; trad. cit., p. 316 : « [L]es contours sont imprécis, les nuances comptent. [...] La vie est une aventure incertaine dans un paysage diffus. » Pour traduire l'incapacité de résumer la vie humaine dans un discours, César la représente sous la forme d'un tableau ; la mort de l'incertain étant aussi la mort du récit.
} 
de ce qui échappe au discours, l'interrompt et par là-même crée une rupture irréparable : devant le tableau, Gaspard en est réduit à balbutier des jeux de mots ${ }^{45}$, César ne parvient pas à exprimer son émotion («En réalité, même maintenant j'ai du mal à l'exprimer avec des mots $\left.^{46} \gg\right)$. Le tableau justifie la persistance de non-dit, en même temps que le mouvement même de l'investigation, et de sa poursuite. Le narrateur du Condottière commente ainsi son rapport au tableau de Messine : «Tu as cherché à l'atteindre et tu as d'abord cru que c'était de l'atteindre qui comptait. Mais seul importait ce mouvement que tu faisais vers lui, ce simple mouvement, cette jetée du corps en avant, ce mouvement de conscience, cette volonté, cet effort. Ce que tu atteindras se trouvera ailleurs ${ }^{47}$. » Le tableau constitue l'investigation comme un récit forcément inachevé - César se défausse en mourant -, lacunaire, indifférent aux prescriptions éthiques comme à celles de la clôture narrative.

L'enquête se constitue donc dans une tension entre deux dispositifs concurrents et complémentaires, un dispositif pictural et un dispositif discursif. Ce dernier vise une certaine maitrise du réel : la mise en récit du passé doit, en le reconstituant, le rendre intelligible et neutraliser sa possible nocivité. En ce sens, l'élucidation du crime dans et par la narration permet une mise en ordre du réel ; les aspects potentiellement inquiétants de l'enquête, ses liens troubles avec le passé, sont surmontés par la clarté de l'exposition. Cette construction vole en éclats avec l'inclusion du tableau dans le récit : loin d'être un dispositif à clarifier, la mise en récit obéit à l'injonction reçue via la contemplation du tableau, celle de l'investigation. Elle n'est pas une tentative de découvrir une solution, mais l'effort par lequel la recherche est promue au rang d'activité indispensable : elle place la justification de l'investigation dans l'investigation elle-même. L'une des figures de l'enquête se constitue donc à partir du réinvestissement et de la transformation des codes du roman policier classique. L'autre fait intervenir les thèmes du secret et de l'exégèse.

\section{La quête et l'exégèse}

L'enquête devient alors «quête », au sens d'une recherche de la cause absente, du secret qui fonde et initie le récit de la recherche ${ }^{48}$. Il n'y a plus de narration d'un événement passé, ni de raisonnement logique à mettre en avant; le mouvement de l'enquête se réalise d'emblée de manière prospective. Le passé peut être l'objet de la quête, mais il ne fait pas l'objet d'une reconstitution ; il est tout entier à construire, comme secret, ou comme énigme, et comme vérité de ce secret. Le récit constitue le secret simultanément dans son existence et dans son impossibilité à être percé. C'est pourquoi l'enquête, là encore, est vouée à l'échec. On reconnaît ce paradigme dans Un cabinet d'amateur, où le secret et la quête constituent les instruments principaux de la manipulation des spectateurs du récit et du lecteur. Le tableau exposé par H. Raffke, sujet de discours contradictoires, est montré comme un objet mystérieux, à partir duquel peut être entreprise une exégèse infinie. Son ensevelissement dans la tombe de son propriétaire en fait un artefact caché, support de fantasmes et de recherches. Il n'est pas seulement constitué en secret ; représentant lui-même les nombreux tableaux - des faux, comme le révèle finalement le narrateur - de la collection Raffke, il renferme une pluralité de secrets à découvrir. Certaines toiles qui y figurent n'ont pas d'attribution certaine, d'autres sont en fait des œuvres perdues - au lecteur de se retrouver dans la masse des noms

\footnotetext{
${ }^{44}$ Dans les deux récits, le tableau est présenté comme l'objet à l'origine de l'acte criminel : César conçoit son meurtre en le voyant pour la première fois, Gaspard tue par frustration de ne pouvoir le falsifier.

${ }^{45}$ Voir par exemple Georges Perec, Le Condottière, éd. cit., p. 112.

${ }^{46}$ Arturo Pérez-Reverte, op. cit., p. 374 : «La verdad es que incluso ahora resulta difícil expresarlo con palabras »; trad. cit., p. 315.

${ }^{47}$ Georges Perec, Le Condottière, éd. cit., p. 201.

${ }^{48}$ Voir Tzvetan Todorov, op. cit., p. $153:$ : Le secret du récit [...] est précisément l'existence d'un secret essentiel [...]. L'essentiel est absent, l'absence est essentielle. »
} 
ou des hypothèses scientifiques que suscitent les tentatives d'identification ou d'attribution entreprises par divers personnages du récit. Surtout, le tableau se représente lui-même, représentant les autres tableaux et se représentant lui-même à nouveau, indéfiniment, mais avec d'infimes variations à déceler à chaque niveau de la mise en abîme. Tout fait secret, tout est signe à déchiffrer, tout peut faire l'objet d'une exégèse. C'est évidemment un moyen de détourner l'attention des objets eux-mêmes - les tableaux de la vente Raffke - et de concentrer la recherche sur ce qui les entoure plutôt que sur leur nature ou leur valeur. Tout incite à la recherche, alors même que c'est en s'y adonnant que les spectateurs du récit, et les lecteurs qui les imitent, sont le mieux mystifiés : les premiers ne voient pas que les tableaux qu'ils achètent sont des faux, les seconds que la majeure partie des peintres et des œuvres évoqués sont des créations fictives. Le secret est ailleurs, dans l'art de la mise en scène du secret.

Dans Le Condottière ${ }^{49}$ et dans Les Onze, l'œuvre d'art est également constituée en un secret qui commande le discours, mais le traitement de ce secret et la construction du discours diffèrent d'un auteur à l'autre. Chez G. Perec, le récit du mystère occulte une vérité autre : le tableau est un faux, les discours sont des faux, le récit obéit au plaisir de la fiction. Cette vérité est inexistante chez P. Michon : la machination existe seule, sans jamais être dénoncée ou commentée par le narrateur. C'est pourquoi, contrairement au Cabinet d'amateur, Les Onze n'offre pas de révélation finale. Plus encore que chez G. Perec, le secret - le tableau des Onze - est créé pour le seul plaisir d'en inventer l'exégèse; le récit se confond avec cette construction. Plus les discours sur Corentin, le «Tiepolo de la Terreur », sont incertains ou contradictoires, plus son existence semble crédible : l'impossible achèvement de l'enquête crée et le secret et le désir de récit. Le tableau n'est «tangible » que par l'effet du discours tenu sur lui, il est tout entier dépendant de lui. D'où le danger des sirènes évoquées par le narrateur et qui détournent le récit de son objet : «Elles aiment mieux les signes d'air que le tangible châssis et la tangible surface peinte [...] qu'on appelle Les Onze, les sirènes. Elles veulent m'empêcher de parler des $O n z e^{50} \gg$. La mise en récit ne fait pas retour sur son objet: elle le produit.

L'enquête est donc un processus narratif: Un cabinet d'amateur est bien l'«histoire d'un tableau », le narrateur michonien se pose comme un conteur qui «racont[e] des histoires ${ }^{51}$ », et l'ami de Gaspard se plaint : «Tu racontes mal ton histoire ${ }^{52} »$. Ce qui importe, ce n'est pas le secret final, c'est la construction d'un récit. L'enquête peut tirer sa valeur d'ellemême parce qu'elle est autant processus de recherche, exploration de la possibilité et du désir de savoir, qu'investigation sur le fonctionnement et les ressources de la littérature elle-même.

\section{Traces et indicialité}

Le recours à des formes policières ou à des structures d'enquête dans la fiction contemporaine s'explique aussi par le fait, que rappelait Carlo Ginzburg dans son article «Spie. Radici di un paradigma indiziario ${ }^{53}$ », que la nécessité de donner sens à l'indice, de faire parler les traces, déclenche un processus narratif. La lecture d'indices, d'une part, permet

\footnotetext{
${ }^{49}$ Voir Georges Perec, Le Condottière, éd. cit., p. $124 s q$. : «Est-ce que je pouvais comprendre ce visage, est-ce que je pouvais comprendre cette maîtrise ?»

${ }^{50}$ Pierre Michon, Les Onze, éd. cit., p. 30.

${ }^{51}$ Ibid., p. 30.

${ }^{52}$ Georges Perec, Le Condottière, éd. cit., p. 141.

${ }^{53}$ Carlo Ginzburg, «Spie. Radici di un paradigma indiziario », dans Aldo Gargani (dir.), Crisi della raggione. Nuovi modelli nel rapporto tra sapere e attività umane, Turin, Einaudi, 1979, traduit sous le titre « Traces. Racines d'un paradigme indiciaire » dans Carlo Ginzburg, Mythes emblèmes traces. Morphologie et histoire, trad. Monique Aymard, Chrisitan Paoloni, Elsa Bonan et Martine Sancini-Vignet, revue par Martin Rueff, Lagrasse, Verdier, « Verdier Poche », 2010.
} 
de retrouver les traces qui signalent une personnalité unique, un style propre : ce qui, dans le tableau, appartient à l'auteur et trahit sa présence ${ }^{54}$. D'autre part, dans les fictions d'enquête, elle est capable de créer un récit entier, rétrospectif, qui délivre la vérité sur le crime, élucidé à partir des indices laissés par le criminel, ou sur un personnage : l'indice fonctionne comme symptôme pour comprendre une personnalité. Ainsi, dans Le Condottière, Gaspard recherche dans les traces laissées sur la toile la vérité sur l'art de Messine et sur sa propre identité. La seconde partie du livre tient autant de l'interrogatoire que de la cure psychanalytique ${ }^{55}:$ la reconstitution du sens du meurtre est inextricablement liée à l'exploration du moi. Dans $U n$ cabinet d'amateur, la question de l'identité du peintre peut sembler moins primordiale, jusqu'à la révélation des alias de Heinrich Kürz, l'auteur du tableau dont on tente de reconstituer la biographie et qui se révèle être Humbert Raffke, le neveu du collectionneur. Dans La tabla de Flandes, l'enquête policière est aussi un processus initiatique, la construction d'une nouvelle identité autonome pour Julia. Dans Les Onze enfin, l'exégèse du tableau et le récit de la vie de Corentin sont un seul et même geste : «Allons, je vois bien qu'à mon tour, quelle que soit ma hâte à bondir vers la fin, [...] il va me falloir raconter à grands traits cette histoire si souvent racontée ${ }^{56}$. » L'enquête concerne autant l'auteur ou l'exégète du tableau qui devient à son tour objet d'enquête, que le tableau lui-même : elle confond ces deux objets et éclaire l'un par l'autre. Le paradigme indiciaire, fondé sur une «méthode d'interprétation basée sur les écarts,

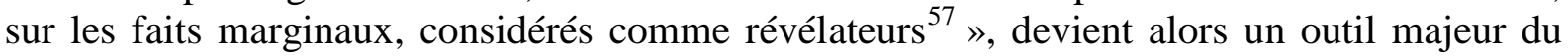
processus de mystification : il est impossible de dire qui, de l'auteur ou du lecteur, est à l'origine de l'indice qui constitue l'écart, impossible de dire si la découverte de l'indice est une trouvaille heuristique ou une extrapolation mystificatrice.

D'autre part, ce que trahit le rapprochement entre le récit policier, l'attribution à un auteur d'un tableau, et la psychanalyse, c'est une mise en équivalence « des symptômes [...], des indices [...], des signes picturaux ${ }^{58} »$. Tous trois supposent le secret : parce que la vérité est invisible ou ne peut être atteinte directement, il faut avoir recours à l'indice pour passer outre les apparences. Or les quatre romans déjouent cette équivalence trompeuse : derrière l'apparence - l'apparition - qu'est le tableau, souvent, il n'y a pas de vérité cachée, et le déchiffrement des signes tourne à vide. D'une part, il n'est absolument pas certain que le tableau puisse être considéré comme un symptôme : les personnages de faussaires viennent opposer un démenti cinglant à la méthode attributionniste. D'autre part, chaque fois que le tableau est envisagé comme un ensemble de signes chiffrés à décoder, la mystification progresse : dans La tabla de Flandes, les enquêteurs se focalisent sur la partie d'échecs rétrospective alors que l'enjeu n'est pas là ${ }^{59}$, ou, dans Les Onze, les conservateurs du Louvre élaborent des explications simplistes : «que ce soient des auteurs, c'est-à-dire des hommes des Lumières, [...] tout cela est marqué sur les pense-bêtes : cela ne se voit pas, sur le tableau ${ }^{60} . »$ Considérer le signe pictural comme un symptôme et un indice, c'est réduire le tableau à un objet appréhensible par le discours, et l'enquête à une structure possédant sa propre solution. En outre, la constitution de l'indice comme indice est avant tout un phénomène de sélection. Si tout fait indice, tout est nivelé, et le récit devient impossible, ou virtuellement infini dans ses interprétations : l'enquête se perd sous le foisonnement du sens.

\footnotetext{
${ }^{54}$ Sur les liens entre enquête, récit et peinture, notamment à travers la promotion du détail pictural comme indice de la personnalité de l'auteur, voir Ibid., p. 220sq.

${ }^{55}$ Voir la préface de Claude Burgelin, à Georges Perec, Le Condottière, éd. cit., p. 14 et p. 21.

${ }^{56}$ Pierre Michon, Les Onze, éd. cit., p. 24.

${ }^{57}$ Carlo Ginzburg, op. cit., p. 230.

${ }^{58}$ Ibid., p. 232.

${ }^{59}$ Voir Jean-Pierre Naugrette, «Arturo Pérez-Reverte : trois fictions policières », dans Denis Mellier et Gilles Menegaldo (dir.), op. cit., p. 159 : «Dans Le Tableau du maître flamand, tout le monde aimerait bien [...] qu'il existe une continuité, une contiguiité des surfaces et des histoires, sinon des époques. ».

${ }^{60}$ Pierre Michon, Les Onze, éd. cit., p. 56.
} 
Or, dans les récits dont il est question ici, le secret est un tableau, le signe est pictural, et l'on retrouve l'un des problèmes fondamentaux de la peinture : de même que dans la peinture tout fait potentiellement signe, tout est potentiellement pertinent, de même l'indice ne prend que le sens qu'on veut bien lui donner, et n'a que l'existence qu'on veut bien lui conférer. Il est porteur de trois dangers : passer inaperçu, être constitué à tort comme indice, être mal interprété. Le lecteur doit éviter à la fois le problème d'une lecture indéfiniment ouverte de l'œuvre, et celui d'une lecture trop naïve car trop littérale. Les auteurs jouent doublement de ces pièges: ils parsèment leur récit d'indices ambigus et proposent des modèles de déchiffrement biaisés. Les indices sont ainsi faux ou déformés (dans Les Onze, les portraits de Corentin n'en sont pas ${ }^{61}$ ), trop ou trop peu présents (dans Un cabinet d'amateur, à la multiplication des variations sur les tableaux dans le tableau répond le manque d'informations sur Kürz ou sur l'histoire de certains tableaux de grande valeur). L'enquête achoppe car l'indice est délibérément mal construit par les auteurs. Elle achoppe également en raison de la nature même de l'indice, qui renvoie immanquablement vers un tout à reconstituer. Or la question de la dialectique du fragment et du tout a également à voir avec le statut du tableau lui-même. Le tableau, dans les récits, fonctionne comme un indice : il tend à être constitué par les personnages ou le narrateur comme la trace d'un acte (d'un meurtre pour La partida de ajedrez), ou d'une identité et d'une volonté (dans Les Onze, où le tableau constitue, d'une certaine manière, un acte politique, dans Le Condottière, avec le faux de Gaspard, miroir de sa conscience, dans Un cabinet d'amateur, dont l'existence fait croire à celle de son prétendu auteur, Kürz). Or il est lui-même le support d'un certain nombre de signes à valeur indiciaire. Les errements de l'enquête viennent donc en partie de la tension entre le détail d'un tableau constitué comme indice et le tout du tableau, entre le tableau considéré comme trace et le tout du secret auquel il renvoie. Les interférences entre les différents niveaux de signification contribuent à la difficulté, pour le lecteur comme pour l'enquêteur, d'assigner un sens à l'œuvre et au récit. Car si l'indice renvoie au tout, à trop se concentrer sur lui, ces deux instances risquent aussi de perdre de vue l'ensemble dans lequel il s'inscrit.

Il y a une différence entre l'indice et le détail : l'indice fonctionne comme un signe qui renvoie à un acte ou à un tout, et à partir duquel on peut élaborer un récit. Il peut être trompeur, mal interprété ; mais en soi, il tend à témoigner d'un ensemble plus large que lui duquel il relève. Le détail fonctionne de manière inverse : il ne renvoie pas au tableau, il est détaché de lui, découpé dans la surface peinte; il tend à s'autonomiser, à acquérir des propriétés et des valeurs propres : «le détail se manifest[e] alors comme un écart ou une résistance par rapport à l'ensemble du tableau; il sembl[e] avoir pour fonction de transmettre une information parcellaire, différente du message global de 1 'œuvre ${ }^{62} »$. Le détail est un surplus de sens ; l'indice pointe vers un sens lacunaire. Le premier propose un récit nouveau à construire ; le second un récit qui soit une reconstitution. L'indice est du côté de l'élucidation ; le détail créé un secret. Ainsi, les détails que sont les variations introduites par Kürz dans le Cabinet d'amateur constituent l'œuvre comme un mystère, se présentent eux-mêmes comme un mystère ; en revanche la partie d'échec, centrale dans la composition de La partida de ajedrez, fonctionne comme un indice, un message chiffré pour élucider le meurtre du chevalier. L'absence même de détails dans le tableau de Messine est vue par Gaspard comme le signe du secret de la maîtrise du peintre italien ${ }^{63}$. C'est lorsque le tableau est constitué comme un indice, en même temps qu'il conserve ses qualités picturales, que le piège se met en place. Ainsi de l'interprétation des Onze prêtée à Michelet : selon le narrateur, l'historien

\footnotetext{
${ }^{61}$ Ibid., p. 14 sq.

${ }^{62}$ Daniel Arasse, Le Détail. Pour une histoire rapprochée de la peinture, Paris, Flammarion, 2011, p. 6.

${ }^{63}$ Voir Georges Perec, Le Condottière, éd. cit., p. 178
} 
« invente » (dans le sens, archéologique, où Daniel Arasse emploie le terme ${ }^{64}$, comme dans son sens classique) dans le tableau des détails qui n'y sont pas et sont censés en révéler le sens profond ; constitués en indices historiques, traces du moment de la commande, ils servent à écrire une Histoire qui tient plus du roman que de la reconstitution scientifique. Superposer le détail à l'indice s'avère ainsi toujours dangereux : en prenant les copies que sont les tableaux dans le tableaux pour les traces des originaux de la collection Raffke, en les traitant donc comme indices de l'existence d'œuvres crues authentiques, et en les constituant dans le même moment comme détails, c'est-à-dire comme écart, dans la mesure où ils innovent par rapport à la stricte reproduction, les spectateur fictifs du Cabinet d'amateur sont complètement mystifiés. Dans Le Condottière, Gaspard traitait habituellement les tableaux qu'il falsifiait selon la méthode attributionniste de Giovanni Morelli : repérage de traits caractéristiques de tel peintre et reconfiguration de ces traits dans une nouvelle toile. En renonçant à chercher dans le tableau des traces, des indices repérables et isolables, et en s'acharnant sur des détails, c'est-à-dire sur des fragments constitués par lui comme détails, dont la subjectivité est assumée et qui se voient par là-même susceptibles d'être récusés aussi rapidement qu'ils ont été constitués ${ }^{65}$, il court, dans sa volonté d'authenticité, droit à l'échec.

Quelle que soit sa forme, l'enquête, dans les textes de notre corpus, ne parvient jamais à proposer de résultat satisfaisant : comme le discours de savoir est contraint de reconnaître ses limites, devant le tableau, l'enquête est mise en échec; elle se perd dans le trop-plein de sens, s'égare, ou au contraire s'avère incapable de nommer le secret, de parvenir au récit cohérent. En cela, elle joue un rôle cognitif tout particulier : si elle n'est pas une structure de résolution, elle propose une méthode de recherche spécifique, à travers laquelle la perception de l'art est radicalement remise en question. La présence du tableau, et le mystère ou l'énigme qu'elle engendre dans les fictions que nous analysons, font du texte un espace intrinsèquement polysémique et trouble : tout détail du récit, quel qu'il soit, peut s'avérer être un signe potentiel qui requiert une interprétation, un indice possible dans le cadre d'une enquête à mener. D'autre part, ils mettent clairement en question le rôle et les possibilités du discours confronté à la fois à l'objet pictural et à l'infini de son interprétation. Ces deux effets sont alors les fondements d'une conception toute nouvelle de la réception des œuvres en même temps qu'un puissant outil pour repenser des frontières génériques traditionnelles.

Loïse LELEVÉ

\footnotetext{
${ }^{64}$ Daniel Arasse, op, cit., p. 7.

${ }^{65}$ Voir Georges Perec, Le Condottière, éd. cit., p. 129 : «Ni l'équilibre ni la logique interne de ton tableau ne poseraient trop de problèmes. [...] Mais ce regard, ces lèvres, ces muscles ? Cette couleur du visage ? Cette sérénité ? [...] Il fallait inventer. Inventer à partir de quoi ?»
} 